\title{
Novel ion drift tube for high-performance ion mobility spectrometers based on a composite material
}

\author{
André Ahrens $^{1}$ (D) Janina Möhle ${ }^{1} \cdot$ Moritz Hitzemann $^{1}$ (D) $\cdot$ Stefan Zimmermann $^{1}$ (D)
}

Received: 26 May 2020 / Revised: 24 June 2020 / Accepted: 26 June 2020 / Published online: 11 July 2020

(C) The Author(s) 2020

\begin{abstract}
Ion mobility spectrometers (IMS) are able to detect ppt $_{\mathrm{V}}$-level concentrations of substances in gasses and in liquids within seconds. Due to the continuous increase in analytical performance and reduction of the instrument size, IMS are established nowadays in a variety of analytical field applications. In order to reduce the manufacturing effort and further enhance their widespread use, we have developed a simple manufacturing process for drift tubes based on a composite material. This composite material consists of alternating layers of metal sheets and insulator material, which are connected to each other in a mechanically stable and gastight manner. Furthermore, this approach allows the production of ion drift tubes in just a few steps from a single piece of material, thus reducing the manufacturing costs and efforts. Here, a drift tube ion mobility spectrometer based on such a composite material is presented. Although its outer dimensions are just $15 \mathrm{~mm} \times 15 \mathrm{~mm}$ in cross section and $57 \mathrm{~mm}$ in length, it has high resolving power of $R_{p}=62$ and detection limits in the $\mathrm{ppt}_{\mathrm{V}}$-range, demonstrated for ethanol and 1,2,3-trichloropropane.
\end{abstract}

Keywords Miniaturized $\cdot$ Ion mobility spectrometer $\cdot$ Drift tube $\cdot$ Composite material $\cdot$ High-performance $\cdot$ Low-cost

\section{Introduction}

Ion mobility spectrometers (IMS) are compact analytical instruments, mainly for the rapid detection of volatile and semivolatile substances at trace levels in the range of $\mathrm{ppt}_{\mathrm{V}}$ (partsper-trillion by volume) within a few seconds [1, 2]. Equipped with electrospray ionization, IMS are also used for measuring larger molecules in liquids [3-5]. Drift tube IMS separate ions in the gas phase under the influence of a homogeneous electric field. A basic setup of a drift tube IMS is shown in Fig. 1. For analysis, the sample is ionized by an ionization source. The ionized sample is then injected into the drift region. Subsequently, driven by the electric field, ions move along the axis of a drift tube through a neutral drift gas and are separated according to their ion mobility. At the end of the ion drift tube, the ions discharge at a detector, often a Faraday plate, and the resulting ion current is recorded over time giving an ion mobility spectrum. At low electric fields, the ion

André Ahrens

ahrens@geml.uni-hannover.de

1 Institute of Electrical Engineering and Measurement Technology, Department of Sensors and Measurement Technology, Leibniz University Hannover, Appelstr. 9A, 30167 Hannover, Germany mobility $K$ is a constant. It is defined as the proportionality factor between the drift velocity $v_{d}$ of the ions and the electric field strength $E$ [6]. As shown in Eq. (1), the ion mobility can thus be derived from the length $L$ of drift tube, the drift voltage $U_{d}$ and the drift time $t_{d}$.

$K=\frac{v_{d}}{E}=\frac{L^{2}}{t_{d} \cdot U_{d}}$

According to the comprehensive theoretical studies of Mason and McDaniel [7], the ion mobility depends mainly on the ion-neutral pair collision cross section and the ion mass. Typically, for comparable results, it is given as reduced ion mobility $K_{0}$ (see Eq. (2)). To correct the influence of a changing number of neutral gas molecules per volume due to pressure or temperature variations, the ion mobility is normalized to the standard temperature $T_{0}=273.15 \mathrm{~K}$ and the standard pressure $p_{0}=1013.25 \mathrm{hPa}[6]$.

$K_{0}=K \cdot \frac{T_{0}}{T} \cdot \frac{p}{p_{0}}$

Two major performance factors of IMS are the detection limits and the resolving power $R_{p}$. Here, the latter is defined according to the time-of-flight mass spectrometry definition [8] as the drift time $t_{d}$ of a peak in an ion mobility spectrum, 
divided by the full peak width $w_{F W H M}$ at half of its maximum (see Eq. (3)).

$R_{p}=\frac{t_{d}}{w_{F W H M}}$

Furthermore, detection limits often are given based on the $3 \sigma$ definition. In particular, the detection limit of a substance is a calculated concentration giving a signal amplitude three times higher than the standard deviation of the zero signal noise.

Due to their fast response times in the range of a few seconds, IMS are used in a variety of applications, including the environmental, medical and, in particular, safety and security sector [9-15]. Monitoring large areas, buildings or crowds of people usually requires a larger number of devices to obtain fast and broad situational awareness. However, acquisition costs increase with the number of devices. One major cost factor is the ion drift tube. In particular, drift tubes often consist of a large number of individual components that have to be manufactured and assembled, such as multiple drift electrodes and insulators. These electrodes are usually arranged at equal distances and are applied to defined electric potentials by means of a resistive voltage divider.

The optimum ratio between drift electrode width and insulating space has already been investigated by Bohnhorst et al. [16]. Another challenge in the construction of ion drift tubes is a gastight design to ensure that no contaminants from outside enter the system and affect the measurement results. To achieve this, either drift electrodes and insulators are stacked and each element is sealed against each other or they are installed in a gastight tube [17-19].

In the past, various designs have been published, how compact drift tube IMS can be manufactured with less effort. These attempts include the processing of drift electrodeinsulator structures on low temperature co-fired ceramics (LTCCs) or standard printed circuit boards (PCBs) [16, 20]. Aiming for less components, IMS based on resistive glass tubes (RGTs) and resistive-coated ceramic tubes have also been reported [21, 22]. Another approach for a small and easy to manufacture drift tube IMS is soldering bended metal strips on a PCB $[23,24]$.

In order to reduce the number of production steps and thus the manufacturing costs of ion drift tubes, we present a novel approach, which allows to manufacture ion drift tubes from a block of a composite material in only a few simple steps [25]. In the production process, structured metal sheets (e.g. stainless steel sheets) and an insulation material are layered alternately. The insulation material can e.g. be a solidified thermoplastic that has adhered to the metal at melted stage or an epoxy resin that bonds the metal when cured. First measurements using an IMS with a composite material based ion drift tube show high resolving power of $R_{p}=62$ and detection limits in the ppt $\mathrm{v}_{\mathrm{V}}$-range for ethanol and 1,2,3-trichloropropane as a model substances.

\section{Experimental}

IMS drift tubes are often composed of a stacked, alternating arrangement of drift electrodes and insulators. With this structure in mind, we have developed a manufacturing approach based on a composite material for ion drift tubes that reduces the high production effort by eliminating the need to manufacture and assemble multiple individual parts. In this approach, the composite material already provides the alternating layer structure. For its production, metal sheets and insulation material are alternately layered and joined in a gastight manner. Subsequently, a bore orientated normal to the alternating layers forms the drift region. A step-by-step sequence of the individual production steps from the very first design to the complete drift tube IMS is depicted in Fig. 2.

\section{Step 1 - material}

First of all, the materials for the later drift tube and hence for the composite material to be produced are determined. The composite material characteristics should meet the requirements for high analytical performance of IMS drift tubes, allow easy machining, and provide sufficient mechanical strength. In detail, the requirements for an ion drift tubes are a gastight construction as well as materials, which are chemically inert and have low outgassing rates. Additionally, the drift electrodes must be electrically conductive and the insulator material must have a sufficient dielectric strength. Thus, stainless steel is particularly suitable for the drift electrodes. The insulation material can consist of any suitable plastic material, which is capable of wetting the metal layers at one time

Fig. 1 Basic setup of a drift tube IMS (left) and an exemplary resulting ion mobility spectrum (right)

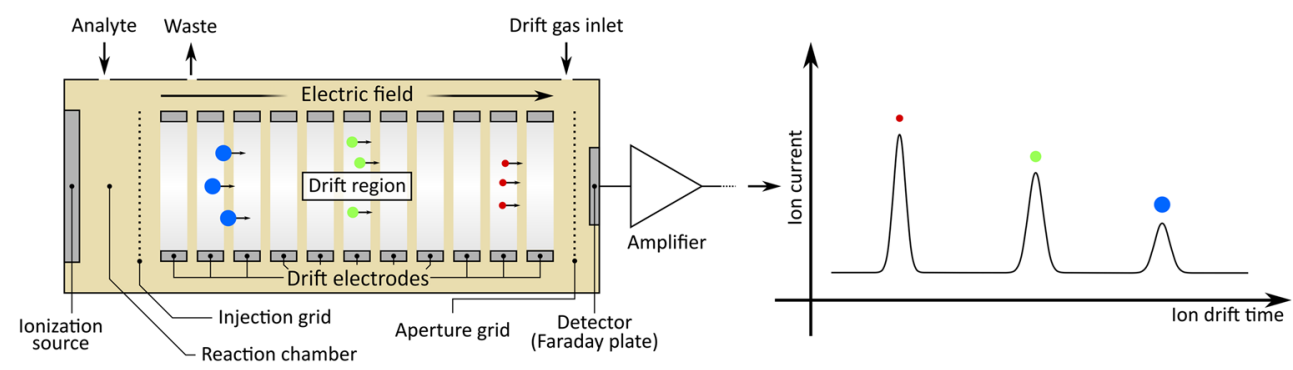



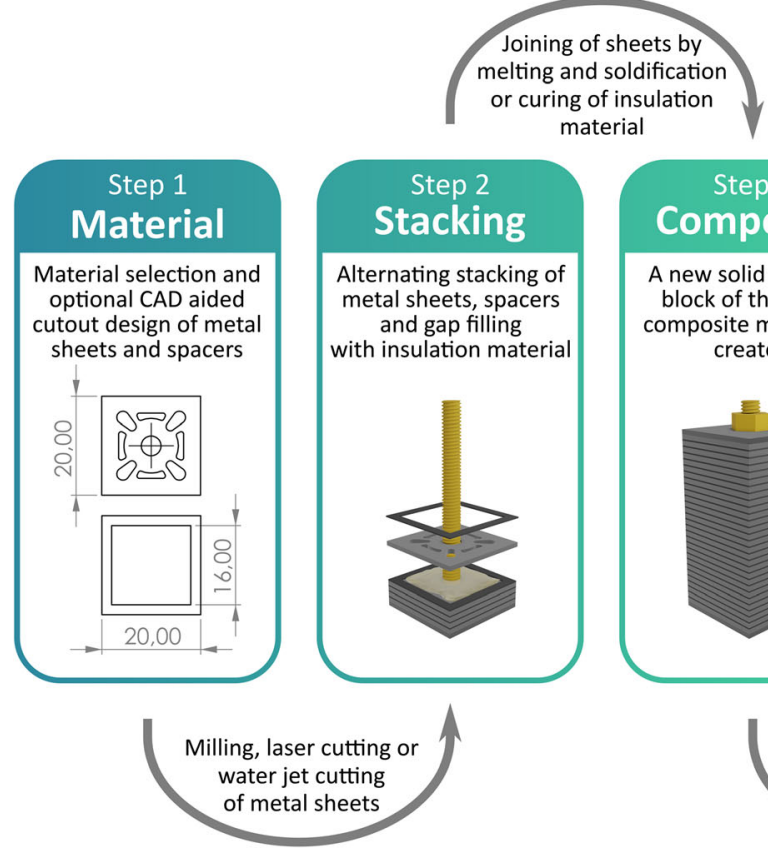
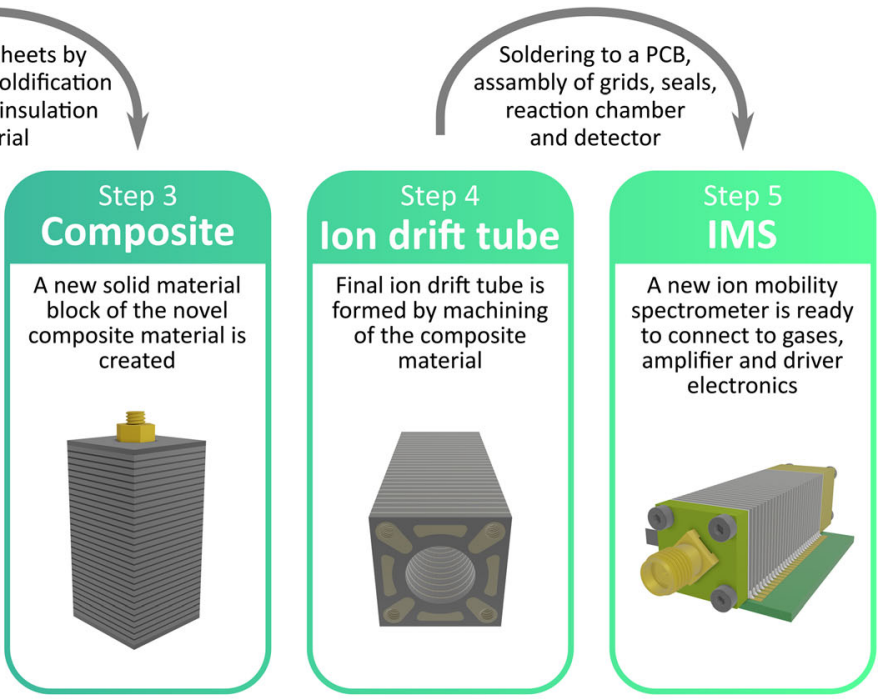

Drilling of drift region, thread forming, milling final outer shape (spacer reoval)

Fig. 2 Production steps to manufacture a composite material based ion drift tube

and thus forming a gastight bond after curing or solidification. Nowadays, for example the high-performance plastic polyether ether ketone (PEEK) can be deposited on stainless steel by flame spraying or electrophoretic deposition. Both materials have been used successfully for IMS manufacturing in the past $[23,26]$. But also less complex to process materials, like commercially available adhesives, are suitable. In this work, a heat-curing adhesive (DELO MONOPOX AD 066 by Delo, Windach, Germany) is used as insulation material.

To support easy machining and to create sufficient mechanical strength, the metal layers can be pre-structured with cutouts. Today, this is done cost-effectively by laser cutting, water jet cutting or milling. Figure 3, left shows a detailed sketch of the cutout pattern on a drift electrode used in this work.

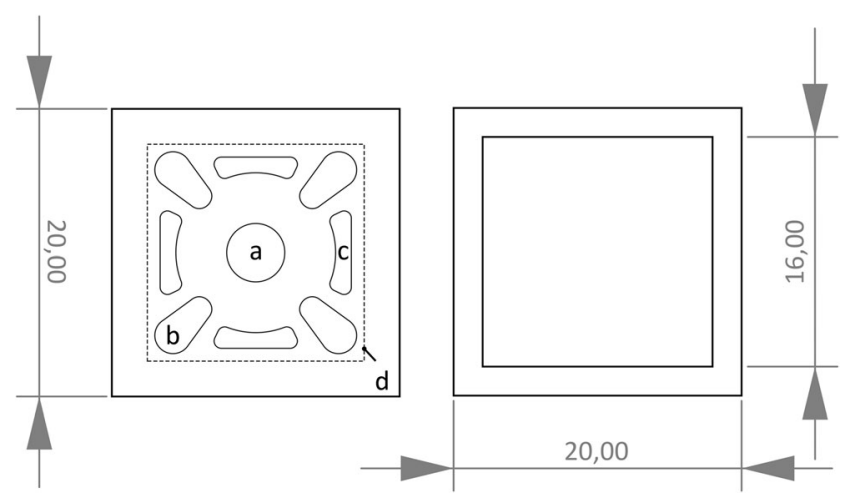

Fig. 3 Left: Detailed layout of a single drift electrode with cutouts for easy layer alignment and drilling (a), thread insulation (b), and mechanical strength incensement (c). The final outer shape $(15 \mathrm{~mm} \times$ $15 \mathrm{~mm}$ ) after machining of the ion the drift tube is given by the dashed line (d). Right: Spacer for insulation layer
Here, the individual cutouts fulfill various tasks. The cutout of the later drift region (see Fig. 3(a)) is necessary to align the metal layers in step 2. If the detector and reaction chamber at a later stage are attached by screws to the drift tube, it must be taken into account that metal screws are unsuitable due to their electrical conductivity. They would short-out the individual drift electrodes during IMS operation and thus, cause an inhomogeneous electric drift field. To avoid this, either plastic screws can be used or the metal layers can be cut out at the points where threads are formed later, as shown in Fig. 3(b). This way the threads are cut completely into insulation material and are thus insulated against the electrodes. Cutouts as depicted in Fig. 3(c) are for increased mechanical strength of the composite material by forming an inner skeleton in order to absorb the forces occurring during the mechanical processing like drilling or milling. The latter type of cutouts also has an additional positive effect on the mechanical strength of the drift tube during operation.

The thickness of a metal layer corresponds to the width of a later drift electrode. Similarly, the thickness of the insulation layer corresponds to the later distance between the drift electrodes. In order to ensure a constant insulation layer thickness during assembly, simple spacers are designed in addition to the drift electrodes, see Fig. 3 (right). The spacers in this work consist of simple frames whose inner cutout is slightly larger than the later outer dimensions of the ion drift tube. Thus, the spacers will be automatically removed in step 4 by milling the outer shape.

In this work 29 stainless steel sheets (type 1.4301 corresponding to DIN EN 10027-2) with a thickness of $1 \mathrm{~mm}$ according to the cutout pattern in Fig. 3 are used to form the 
composite material. The spacers are also made of stainless steel with a thickness of $0.3 \mathrm{~mm}$. All stainless steel parts are laser cut and purchased from Laserhub GmbH, Stuttgart, Germany.

\section{Step 2 - stacking}

After cleaning of all metal layers with isopropyl alcohol to ensure good adhesion of the insulation material, the metal layers and spacers are alternately threaded onto a threaded rod. In the end of this step, the threaded rod is used for pressing the composite material block together during the curing process. The gaps formed by the cutouts in each metal layer are periodically filled with insulation material. Since the insulation material used is liquid during processing, it is recommended to provide the first and the last metal layer without any cutouts than the one in center. These two additional layers will be removed in step 4. The leakage of liquid insulation material at the sides of the block is prevented by the spacers due to their frame shape forming a chamber. After positioning the last layer, the block of material is fixed to the threaded rod with two nuts. Due to this pressure, the final insulation layers are approximately at the same thickness as the spacers. At last, curing is carried out for $60 \mathrm{~min}$ at $100{ }^{\circ} \mathrm{C}$ in an oven. When using a thermoplastic insulation material, the procedure is more complex, since metal layers and insulation material must have the right temperatures for a reliable bound and melted plastics are more difficult to handle.

\section{Step 3 - composite}

A material block of the composite material is created. Although the process for a single drift tube is described in this paper, the manufacturing process can be easily transferred to several drift tubes in parallel. For this, only the metal layers and spacers have to be adapted during the design phase in step 1. As an example, a metal layer composed of four by four parallel drift tubes is shown in Fig. 4. This illustrates the massive reduction in manufacturing effort, since steps 1 to 3 are the same for a single one or sixteen drift tubes at once.

\section{Step 4 - ion drift tube}

After the insulation material is cured or solidified, the composite material block is machined. In detail, the drift region of the ion drift tube is formed by drilling a hole along the axis of the threaded rod, which thus is removed at the same time. Additionally, threads are cut for mounting the reaction chamber and detector as well as a hole for the waste-capillary is drilled. Also the auxiliary structures such as spacers and first and last metal layer (see step 2) are removed. If several parallel drift tubes are manufactured (as depicted in Fig. 4), they will be separated in this step.

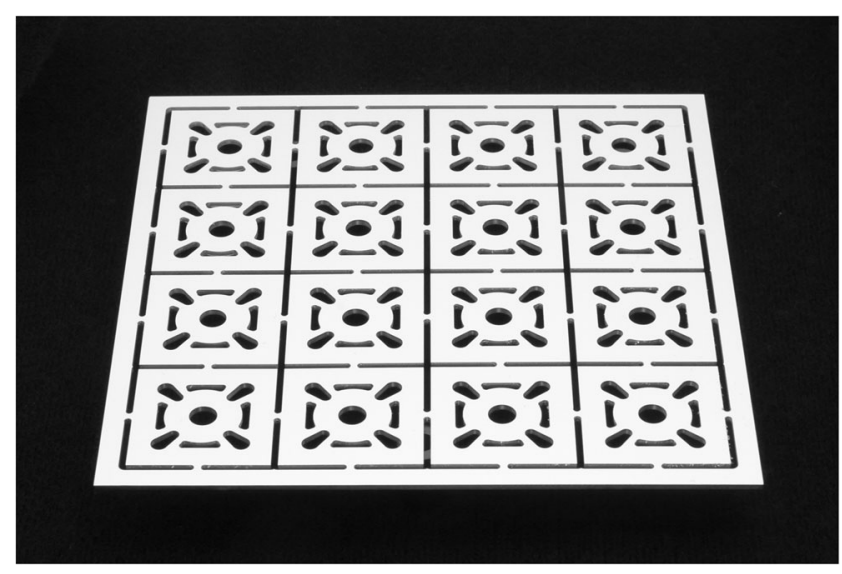

Fig. 4 Exemplary milled metal layer (1 $\mathrm{mm}$ in thickness) with a pattern of four times four single drift electrodes, forming sixteen ion drift tubes when processed to a composite material block

\section{Step 5 - IMS}

In this step, a detector and a reaction chamber necessary for the operation of a drift tube IMS are assembled. Finally, Fig. 5 shows an exemplary IMS with a drift tube manufactured of such a composite material, a PEEK reaction chamber and a detector based on a PCB. The entire IMS has outer dimensions of only $15 \mathrm{~mm} \times 15 \mathrm{~mm}$ in cross-section and $57 \mathrm{~mm}$ in length.

The ionization source, used in this work is a tritium source with an activity of $130 \mathrm{MBq}$. Once a gaseous sample is ionized, the ion cloud is transferred from the reaction chamber to the drift region by a field switching shutter [27]. For this, an injection field of $282.5 \mathrm{~V} / \mathrm{mm}$ and a blocking field of $-0.25 \mathrm{~V} /$ $\mathrm{mm}$ and $0.45 \mathrm{~V} / \mathrm{mm}$ in positive ion mode and in negative ion mode is used, respectively. The drift length of the IMS is $L=38.1 \mathrm{~mm}$ by counting 29 layers. With a metal layer thickness of $1 \mathrm{~mm}$ and an insulation layer thickness of $0.3 \mathrm{~mm}$ a drift length of $37.4 \mathrm{~mm}$ is expected. Nevertheless, the difference is caused by manufacturing tolerances of the composite material block. The detector, a Faraday plate, at the end of the

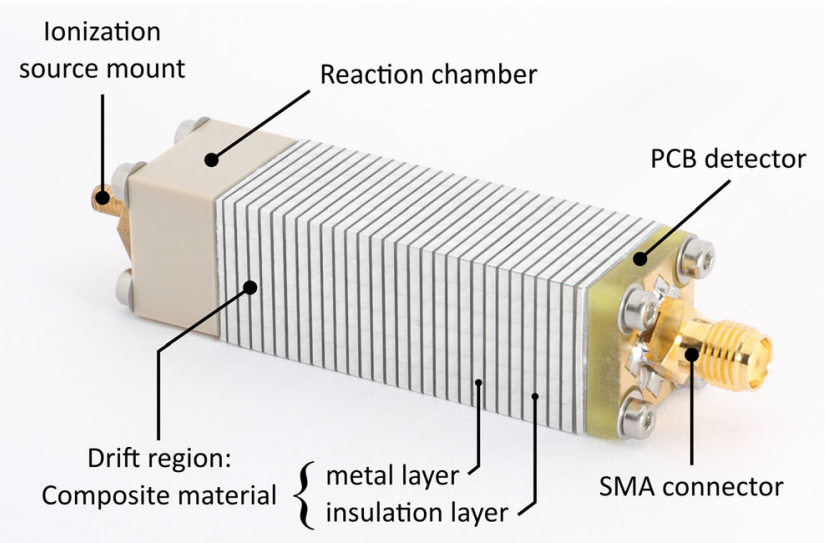

Fig. 5 Compact IMS with a drift tube made from a composite material (DELO MONOPOX AD 066 as insulation material). Outer dimensions are only $15 \mathrm{~mm} \times 15 \mathrm{~mm}$ in cross-section and $57 \mathrm{~mm}$ in length 
drift region is made from a common PCB and has been taken from an earlier measurement setup, like the reaction chamber with slight modifications and also the ionization source [23].

The drift region is continuously flushed by $120 \mathrm{ml} / \mathrm{min}$ (mass flow reference conditions $20^{\circ} \mathrm{C}$ and $1013.25 \mathrm{hPa}$ ) purified, dry (dew point of $-90{ }^{\circ} \mathrm{C}$ ) air as drift gas. The sample carrier gas flow is $10 \mathrm{ml} / \mathrm{min}$. A gas mixing system connected to a permeation oven (Dynacalibrator Model 150 by Vici Metronics Inc., Poulsbo, WA, USA) is used to set the sample concentration in the sample carrier gas. This system allows further dilution of defined fractions of the permeation oven gas flow with purified gas in a controlled manner to obtain the desired concentrations. Mass flow controllers (EL-FLOW SELECT by Bronkhorst, Kamen, Germany) are used to control the gas flows. The permeation oven is loaded with homemade permeation tubes filled with chemicals purchased from Sigma-Aldrich with a purity of $>99 \%$. The masses of the permeation tubes are monitored over days up to weeks, as there is just a slight mass reduction over time. Due to the very low concentration of compounds in the sample carrier gas, surface adsorption effects need to be considered. Depending on the adsorption characteristics of the respective compound, the setting times for each concentration ranges from a few minutes for highly volatile substances to several hours and even days for sticky, semi-volatile substances. Further errors in gas dosing result from the errors of the mass flow controllers and the scale to measure the mass of the permeation tubes.

Since the drift electrodes are accessible from the outside due to the composite material in this setup, the individual electrodes are electrically connected through direct soldering on a PCB containing a resistive voltage divider. This easy electrical connection to the electronic driver circuit leads to a drift tube integration similar to surface mounted device

Table 1 Dimensions and operating parameters of the IMS

\begin{tabular}{ll}
\hline Parameter & Value \\
\hline Ionization source & Tritium \\
Ionization source activity & $130 \mathrm{MBq}$ \\
Ionization source active diameter & $10 \mathrm{~mm}$ \\
Field switching injection field & $282.5 \mathrm{~V} / \mathrm{mm}$ \\
Field switching blocking field & $-0.25 \mathrm{~V} / \mathrm{mm}$ and $0.45 \mathrm{~V} / \mathrm{mm}$ \\
Drift length $L$ & $38.1 \mathrm{~mm}$ \\
Drift region diameter & $8 \mathrm{~mm}$ \\
Drift region field & $63.5 \mathrm{~V} / \mathrm{mm}$ \\
Repetition rate & $40 \mathrm{~Hz}$ \\
Drift gas flow & $120 \mathrm{ml} / \mathrm{min}$ \\
Sample carrier gas flow & $10 \mathrm{ml} / \mathrm{min}$ \\
Dew point of drift gas and sample gas & $-90{ }^{\circ} \mathrm{C}$ \\
Inner IMS pressure $p$ & $996 \mathrm{hPa}-1046 \mathrm{hPa}$ \\
IMS operating temperature $T$ & $23{ }^{\circ} \mathrm{C}-25^{\circ} \mathrm{C}$ \\
\hline
\end{tabular}
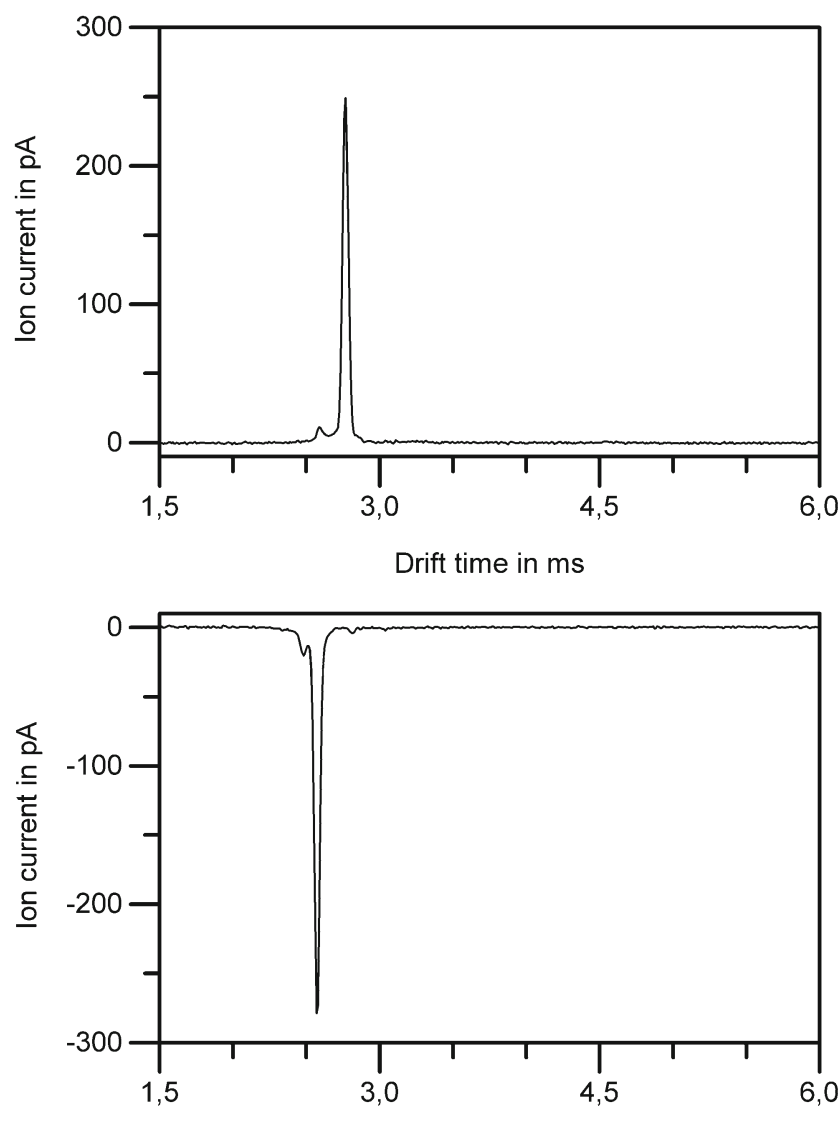

Drift time in $\mathrm{ms}$

Fig. 6 Positive (top) and negative (bottom) reactant ion Peak (RIP) in purified, dry (dew point $-90^{\circ} \mathrm{C}$ ) air. Measuring parameters see Table 1 (and $p=996 \mathrm{hPa}, T=25^{\circ} \mathrm{C}$ )

(SMD) components. In addition, the exposed drift electrodes offer direct heat transfer from an outer heat source into the drift tube if required. However, this has been omitted for measurements in this work.

The dimensions and operating parameters of the IMS are summarized in Table 1.

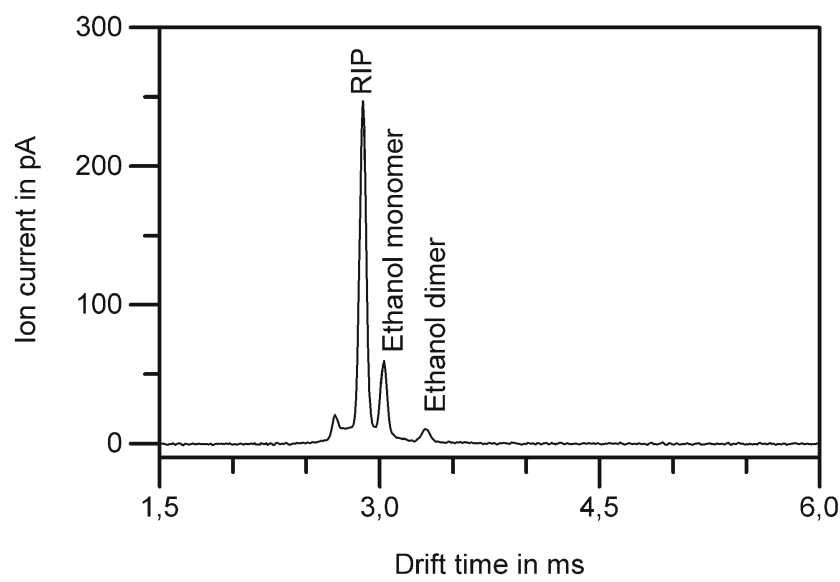

Fig. 7 Ion mobility spectrum of $530 \mathrm{ppt}_{\mathrm{V}}$ ethanol in purified, dry air. Parameters see Table 1 (and $p=1046 \mathrm{hPa}, T=23{ }^{\circ} \mathrm{C}$ ); monomer $K_{0}=$ $1.89 \mathrm{~cm}^{2} /(\mathrm{Vs})$; dimer $K_{0}=1.73 \mathrm{~cm}^{2} /(\mathrm{Vs})$ 
Fig. 8 Calibration curve of ethanol monomer (left) and 1,2,3trichloropropane (right) in purified dry air. Parameters see Table 1 (and $p=1046 \mathrm{hPa}, T=$ $23{ }^{\circ} \mathrm{C}$ )

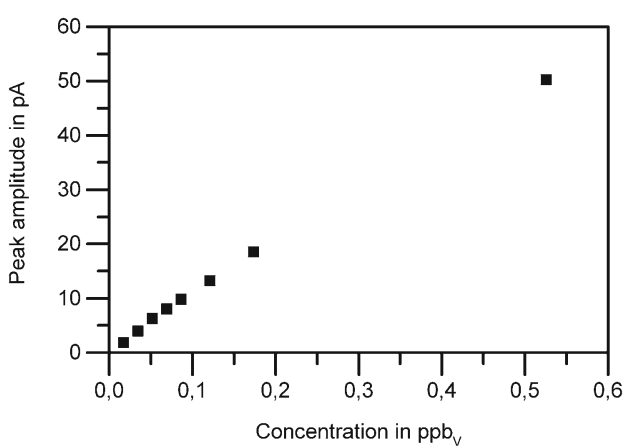

\section{Results and discussion}

To characterize the drift tube, the resolving power and the detection limits of ethanol and 1,2,3-trichloropropane are determined. In the following measurements, purified dry air (dew point of $-90^{\circ} \mathrm{C}$ ) is used as drift and sample carrier gas.

The resolving power of the IMS is determined based on the positive reactant ion peak (RIP) mainly consisting of protonated water clusters $H^{+}\left(\mathrm{H}_{2} \mathrm{O}\right)_{n}$ under the used operating conditions [6, $28]$. Figure 6 top shows the ion mobility spectrum of purified dry air in positive ion mode. The resolving power of the dominating positive RIP at a drift time of $t_{d}=2.77 \mathrm{~ms}$ is $R_{P}=62$. The reduced ion mobility of the positive RIP is $K_{0}=1.95 \mathrm{~cm}^{2} /(\mathrm{Vs})$.

Similarly, Fig. 6 bottom shows the ion mobility spectrum of purified dry air in negative ion mode. The resolving power of the dominating negative RIP at a drift time of $t_{d}=2.58 \mathrm{~ms}$ is $R_{P}=60$. Its reduced ion mobility is $K_{0}=2.1 \mathrm{~cm}^{2} /(\mathrm{Vs})$.

As mentioned above, ethanol and 1,2,3-trichloropropane have been used as exemplary substances. An ion mobility spectrum of $530 \mathrm{ppt}_{\mathrm{V}}$ ethanol is shown in Fig. 7. To determine the detection limit of ethanol, the peak amplitude of the ethanol monomer is recorded while varying the concentration of ethanol in the sample gas between 20 ppt $_{\mathrm{V}}$ and $530 \mathrm{ppt}_{\mathrm{V}}$. In Fig. 8 left, the measured peak amplitudes are plotted against the concentration of ethanol in the sample gas.

Taken into account a noise level of $\sigma=1.8 \mathrm{pA}$ for an averaging time of one second, detection limits of $50 \mathrm{ppt}_{\mathrm{V}}$ and 550 $\mathrm{ppb}_{\mathrm{V}}$ for the ethanol monomer and dimer are calculated based on the $3 \sigma$ definition. The calibration curve for the ethanol monomer is shown in Fig. 8 (left).

Similarly, the detection limit of 1,2,3-trichloropropane is determined. The calibration curve of 1,2,3-trichloropropane is shown in Fig. 8 (right). Again, for an averaging time of one second, the detection limit of 1,2,3-trichloropropane is calculated to $200 \mathrm{ppt}_{\mathrm{V}}$.

\section{Conclusion}

In this work, we presented a novel approach for easy and costreduced manufacturing of drift tubes for IMS based on a composite material consisting of alternating layers of metal sheets and insulator material, which are connected in a mechanically stable and gastight manner. As a first benchmark, a high-performance drift tube IMS with $38.1 \mathrm{~mm}$ drift length, only $15 \mathrm{~mm} \times 15 \mathrm{~mm}$ cross-section and $57 \mathrm{~mm}$ in overall length (outer dimensions) has been realized using such a composite material. This demonstrator shows high resolving power of $R_{p}=62$ and detection limits for the ethanol monomer of $50 \mathrm{ppt}_{\mathrm{V}}$ and the ethanol dimer of $550 \mathrm{ppt}_{\mathrm{V}}$, measured in purified dry air with an averaging time of one second. Additionally, a detection limit of $200 \mathrm{ppt}_{\mathrm{V}}$ for 1,2,3trichloropropane has been determined, again measured in $\mathrm{pu}-$ rified dry air with an averaging time of one second. The proposed composite material allows the production of a single drift tube, but also the parallel production of several drift tubes in one process.

Funding Information Open Access funding provided by Projekt DEAL.

\section{Compliance with ethical standards}

Conflict of interest The authors declare that they have no conflict of interest.

Open Access This article is licensed under a Creative Commons Attribution 4.0 International License, which permits use, sharing, adaptation, distribution and reproduction in any medium or format, as long as you give appropriate credit to the original author(s) and the source, provide a link to the Creative Commons licence, and indicate if changes were made. The images or other third party material in this article are included in the article's Creative Commons licence, unless indicated otherwise in a credit line to the material. If material is not included in the article's Creative Commons licence and your intended use is not permitted by statutory regulation or exceeds the permitted use, you will need to obtain permission directly from the copyright holder. To view a copy of this licence, visit http://creativecommons.org/licenses/by/4.0/.

\section{References}

1. Kirk AT, Küddelsmann MJ, Bohnhorst A, Lippmann M, Zimmermann S, Kirk AT, Kueddelsmann MJ (2020) Improving ion mobility spectrometer sensitivity through the extended field switching ion shutter. Anal Chem 92(7):4838-4847 
2. Bunert E, Reinecke T, Kirk AT, Bohnhorst A, Zimmermann S (2018) Ion mobility spectrometer with orthogonal X-ray source for increased sensitivity. Talanta 185:537-541

3. Kanu AB, Hampikian G, Brandt SD, Hill HH (2010) Ribonucleotide and ribonucleoside determination by ambient pressure ion mobility spectrometry. Anal Chim Acta 658(1):91-97

4. Bramwell CJ, Colgrave ML, Creaser CS, Dennis R (2002) Development and evaluation of a nano-electrospray ionisation source for atmospheric pressure ion mobility spectrometry. Analyst 127(11):1467-1470

5. Shumate CB, Hill HH (1989) Coronaspray nebulization and ionization of liquid samples for ion mobility spectrometry. Anal Chem 61(6):601-606

6. Eiceman GA, Karpas Z, Hill HH (2013) Ion mobility spectrometry. CRC Press, Boca Raton

7. Mason EA, McDaniel EW (1988) Transport properties of ions in gases. Wiley-VCH Verlag GmbH \& Co. KGaA, Weinheim, FRG

8. Nič M, Jirát J, Košata B, Jenkins A, McNaught A (2009) IUPAC compendium of chemical terminology. IUPAC, eResearch Triagle Park

9. Eiceman GA, Stone JA (2004) Peer reviewed: ion mobility spectrometers in National Defense. Anal Chem 76:390A-397A

10. Allers M, Langejürgen J, Gaida A, Holz O, Schuchardt S, Hohlfeld JM, Zimmermann S (2016) Measurement of exhaled volatile organic compounds from patients with chronic obstructive pulmonary disease (COPD) using closed gas loop GC-IMS and GC-APCIMS. J Breath Res 10(2):26004

11. Perl T, Carstens E, Hirn A, Quintel M, Vautz W, Nolte J, Jünger M (2009) Determination of serum propofol concentrations by breath analysis using ion mobility spectrometry. Br J Anaesth 103(6):822827

12. Tam M, Hill HH (2004) Secondary electrospray ionization-ion mobility spectrometry for explosive vapor detection. Anal Chem 76(10):2741-2747

13. Ewing RG, Atkinson DA, Eiceman GA, Ewing GJ (2001) A critical review of ion mobility spectrometry for the detection of explosives and explosive related compounds. Talanta 54(3):515-529

14. Arnanthigo Y, Anttalainen O, Safaei Z, Sillanpää M (2016) Snifftesting for indoor air contaminants from new buildings environment detecting by aspiration-type ion mobility spectrometry. Int J Ion Mobil Spectrom 19(1):15-30

15. Dion HM, Ackerman LK, Hill J, Herbert H (2002) Detection of inorganic ions from water by electrospray ionization-ion mobility spectrometry. Talanta 57(6):1161-1171
16. Bohnhorst A, Kirk AT, Zimmermann S (2016) Simulation aided design of a low cost ion mobility spectrometer based on printed circuit boards. Int J Ion Mobil Spectrom 19(2-3):167-174

17. Hauck BC, Siems WF, Harden CS, McHugh VM, Hill HH (2017) Construction and evaluation of a hermetically sealed accurate ion mobility instrument. Int J Ion Mobil Spectrom 76:390A

18. Babis JS, Sperline RP, Knight AK, Jones DA, Gresham CA, Denton MB (2009) Performance evaluation of a miniature ion mobility spectrometer drift cell for application in hand-held explosives detection ion mobility spectrometers. Anal Bioanal Chem 395(2): 411-419

19. Kirk AT, Allers M, Cochems P, Langejürgen J, Zimmermann S (2013) A compact high resolution ion mobility spectrometer for fast trace gas analysis. Analyst 138(18):5200-5207

20. Pfeifer KB, Rohde SB, Peterson KA, Rumpf AN (2004) Development of rolled miniature drift tubes using low temperature co- fired ceramics (LTCC). Int J Ion Mobil Spectrom 7:52-58

21. Kwasnik M, Fuhrer K, Gonin M, Barbeau K, Fernandez FM (2007) Performance, resolving power, and radial ion distributions of a prototype nanoelectrospray ionization resistive glass atmospheric pressure ion mobility spectrometer. Anal Chem 79(20):7782-7791

22. Carrico JP, Sickenberger DW, Spangler GE, Vora KN (1983) Simple electrode design for ion mobility spectrometer. J Phys E: Sci Instrum 16(11):1058-1062

23. Ahrens A, Hitzemann M, Zimmermann S (2019) Miniaturized high-performance drift tube ion mobility spectrometer. Int J Ion Mobil Spectrom 22(2):77-83

24. Bradshaw RFD (20.08.1996) Drift Chambers. EP0920619B1

25. Zimmermann S, Ahrens A (29.01.2016) Ionentransportröhre und Verfahren zu deren Herstellung. DE 102016101598 B4

26. Langejürgen J, Allers M, Oermann J, Kirk AT, Zimmermann S (2014) Quantitative detection of benzene in toluene- and xylenerich atmospheres using high-kinetic-energy ion mobility spectrometry (IMS). Anal Chem 86(23):11841-11846

27. Kirk AT, Zimmermann S (2014) Bradbury-Nielsen vs. Field switching shutters for high resoluetion drift tube ion mobility spectrometers. Int J Ion Mobil Spectrom 17:131-137

28. Kebarle P, Searles SK, Zolla A, Scarborough J, Arshadi M (1967) Solvation of the hydrogen ion by water molecules in the gas phase. Heats and entropies of solvation of individual reactions. $\mathrm{H}+$ (H2O)n-1 + H2O .fwdarw. H+(H2O)n. J Am Chem Soc 89(25): 6393-6399

Publisher's note Springer Nature remains neutral with regard to jurisdictional claims in published maps and institutional affiliations. 